\title{
ANTI-PERIODIC BOUNDARY VALUE PROBLEMS FOR NONLINEAR HIGHER ORDER IMPULSIVE DIFFERENTIAL EQUATIONS
}

\author{
Yuji Liu and Zhanji Gui
}

\begin{abstract}
This paper is concerned with the anti-periodic boundary value problems for nonlinear higher order impulsive differential equations

$$
\left\{\begin{aligned}
x^{(n)}(t) & =f\left(t, x(t), x^{\prime}(t), \cdots, x^{(n-1)}(t)\right), t \in[0, T], t \neq t_{k}, k=1, \cdots, p, \\
\Delta x^{(i)}\left(t_{k}\right) & =I_{i, k}\left(x\left(t_{k}\right), x^{\prime}\left(t_{k}\right), \cdots, x^{(n-1)}\left(t_{k}\right)\right), k=1, \cdots, p, i=0, \cdots, n-1, \\
x^{(i)}(0) & =-x^{(i)}(T), i=0, \cdots, n-1 .
\end{aligned}\right.
$$
\end{abstract}

We obtain sufficient conditions for the existence of at least one solution. Examples are presented to illustrate the main results.

\section{INTRODUCTION}

There exist many papers concerned with the solvability of periodic boundary value problems or the existence of periodic solutions for higher order ordinary differential equations with or without impulses effects.

For examples, in $[1,2]$, the existence of periodic solutions of the the equation

$$
x^{(n)}(t)=f\left(t, x(t), x^{\prime}(t), \cdots, x^{(n-1)}(t)\right)
$$

has been studied by many authors [1-7] under a variety of conditions on $f$. The authors proved that equation (1) has at least one periodic solution if some conditions

Received November 21, 2005, accepted September 20, 2006.

Communicated by Kening Lu.

2000 Mathematics Subject Classification: 34B10, 34B15.

Key words and phrases: Anti-Periodic boundary value problem, Higher order impulsive differential equation, Fixed-point theorem, Growth condition.

The author was supported by the Science Foundation of Hunan Province and partially by the National Natural Sciences Foundation of P. R. China. 
imposed on $f$ are satisfied. In [3-7], the authors studied the existence of periodic solutions of the following differential equations

$$
x^{(2 n)}(t)+\sum_{i=1}^{n-1} a_{i} x^{(2 i)}(t)+f(t, x(t))=0, \quad t \in R,
$$

and

$$
x^{(2 n+1)}(t)+\sum_{i=1}^{n} a_{i} x^{(2 i-1)}(t)+f(t, x(t))=0, \quad t \in R,
$$

respectively. Equations (2) and (3) are special cases of equation (1).

In paper [12-15], the problems

$$
\left\{\begin{aligned}
x^{\prime}(t) & =f(t, x(t)), t \in[0, T], t \neq t_{k}, k=1, \cdots, m, \\
\Delta x\left(t_{k}\right) & =I_{k}\left(x\left(t_{k}\right)\right), k=1, \cdots, m \\
x(0) & =x(T)
\end{aligned}\right.
$$

and

$$
\left\{\begin{aligned}
x^{\prime \prime}(t) & =f\left(t, x(t), x^{\prime}(t)\right), t \in[0, T], t \neq t_{k}, k=1, \cdots, m, \\
\Delta x\left(t_{k}\right) & =I_{k}\left(x\left(t_{k}\right), x^{\prime}\left(t_{k}\right)\right), k=1, \cdots, m \\
\Delta x^{\prime}\left(t_{k}\right) & =I_{k}\left(x\left(t_{k}\right), x^{\prime}\left(t_{k}\right)\right), k=1, \cdots, m \\
x(0) & =x(T), x^{\prime}(0)=x^{\prime}(T)
\end{aligned}\right.
$$

were studied. The existence results for these problems were established by using lower and upper solutions methods. Some recent studies on the existence of periodic solutions and their stability for functional or ordinary differential equations, which are arise in many applications, with or without impulses effects can be found in [34-44] and the references therein. The general theory of impulsive differential equations (IDE) and systems can be found in [27, 31-33].

The existence of solutions for anti-periodic boundary value problems for first order impulsive ordinary differential equations was studied in [8-11, 16, 21]. When the impulses are absent, i.e., $I_{k}=0$ for $k=1, \cdots, m$, anti-periodic boundary value problems for first order ordinary differential equations were studied in [9-11], anti-periodic problems for nonlinear differential equations in Hilbert spaces, and for nonlinear evolution equations have been studied in papers [20,28-30]. Also, antiperiodic boundary conditions for partial differential equations and abstract differential equations are considered in [17-19]. Anti-periodic boundary value problems for higher order differential equations are studied in [22]. Notice that anti-periodic 
boundary value problems appear in physics in a variety of situations, see, for example, [19-21, 24-26].

In paper [16], Luo, Shen and Nieto studied the problem

$$
\left\{\begin{aligned}
x^{\prime}(t) & =f(t, x(t)), t \in[0, T], t \neq t_{k}, k=1, \cdots, m, \\
\Delta x\left(t_{k}\right) & =I_{k}\left(x\left(t_{k}\right)\right), k=1, \cdots, m, \\
x(0) & =-x(T),
\end{aligned}\right.
$$

and the following results were obtained.

Theorem. Suppose $\lambda>0$. Assume that there is function $\psi:[0,+\infty) \rightarrow$ $(0,+\infty)$ and a function $\rho \in L^{1}([0, T])$ with

$$
|f(t, x)+\lambda x| \leq \rho(t) \psi(|x|),
$$

and there exist $b_{k} \geq 0$ such that

$$
\left|I_{k}(x)\right| \leq b_{k}|x| \text { and } \sum_{k=1}^{m} b_{k}<1+e^{-\lambda T} .
$$

Furthermore, suppose

$$
\sup _{c \geq 0} \frac{c}{\psi(c)}>\frac{\|\rho\|_{L^{1}}}{1+e^{-\lambda T}-\sum_{k=1}^{m} b_{k}} .
$$

Then (6) has at least one solution.

Under the assumptions

$$
f(t, u)-f(t, v) \geq-\lambda(u-v)+M(u-v)
$$

and that there are a pair of coupled lower and upper solutions for (4), and $I_{k}$ are nondecreasing, and other assumptions, the existence result was also proved by authors in $[8,10]$ using lower and upper solutions methods and monotone iterative technique.

We note that equation (7) or (8) implies that $f(t, x)$ grows at most linearly in $x$. So the problem have not been solved when $f(t, x)$ is supper for $x$. Furthermore, there exist no paper concerned with the solvability of anti-periodic problems for higher order impulsive differential equations.

In this paper, we are concerned with the existence of solutions of the anti-periodic boundary value problems for nonlinear impulsive functional differential equations 
(9)

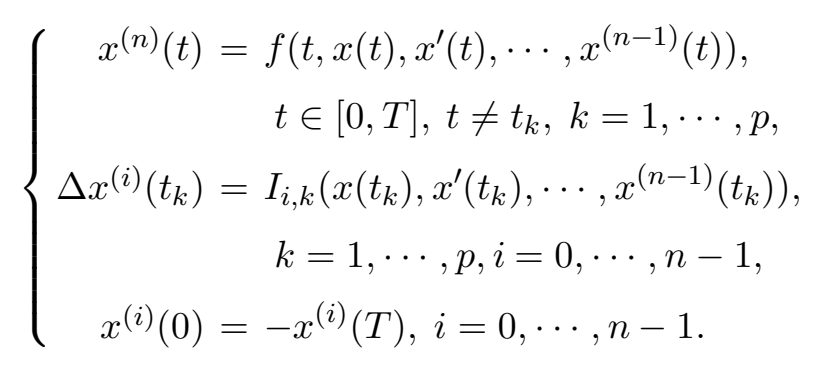

where $n \geq 2, T>0,0<t_{1}<\cdots<t_{m}<T$ are constants, $f$ is an impulsive Carathedeodory function, $I_{i, k}$ are continuous functions. The purpose is to establish existence results for solutions of (9). We do not require the assumption that $f$ is at most linear nor $I_{i, k}$ are nondecreasing. Finally, some examples illustrate the main results.

\section{Main Results and Proofs}

In this section, we establish the main results. To define solutions of (9), we introduce the Banach space.

Let $u: J=[0, T] \rightarrow R$, and $0=t_{0}<t_{1}<\cdots<t_{p}<t_{p+1}=T$, for $k=0, \cdots, p$, define the function $u_{k}:\left(t_{k}, t_{k+1}\right) \rightarrow R$ by $u_{k}(t)=u(t)$. We will use the following Banach space

$$
X=\left\{\begin{array}{l}
u: J \rightarrow R, u_{k}^{(i)} \in L^{1}\left(t_{k}, t_{k+1}\right), k=0, \cdots, p, i=0, \cdots, n-1, \\
\text { the limits exist } \lim _{t \rightarrow t_{k}^{-}} x(t)=x\left(t_{k}\right), \\
\lim _{t \rightarrow t_{k}^{+}} x(t), \lim _{t \rightarrow 0^{+}} x(t)=x(0), \lim _{t \rightarrow T^{-}} x(t)=-x(T)
\end{array}\right\}
$$

and

$$
Y=\left\{u \in X, u_{k} \in W^{1,1}\left(t_{k}, t_{k+1}\right), k=0, \cdots, p\right\} \times R^{p n}
$$

with the norms

$$
\|u\|_{X}=\max \left\{\sup _{t \in\left(t_{k}, t_{k+1}\right)}\left|u_{k}(t)\right|, k=0, \cdots, p\right\}
$$

for $u \in X$ and

$$
\|y\|_{Y}=\max \left\{\left\|u_{k}\right\|_{W^{1,1}\left(t_{k}, t_{k+1}\right)}, k=0, \cdots, p, \max _{1 \leq k \leq p}\left\{\left|x_{k}\right|\right\}\right\}
$$

for $y=\left\{u, x_{1}, \cdots, x_{p n}\right\} \in Y$.

A function $F$ is an impulsive Carathedeodory function if

$* F\left(\bullet, u_{0}, u_{1}, \cdots, u_{n}\right)$ is measurable for each $u \in R$; 
$* F(t, \bullet, \cdots, \bullet)$ is continuous for a.e. $t \in J \backslash\left\{t_{1}, \cdots, t_{p}\right\}$;

* for each $r>0$ there is $h_{r} \in L^{1}(J)$ so that

$$
\left|F\left(t, u_{0}, u_{1}, \cdots, u_{n}\right)\right| \leq l_{r}(t) \text {, a.e.t } \in J \backslash\left\{t_{1}, \cdots, t_{p}\right\}
$$

and every $u$ satisfying $\left\|\left(u_{0}, u_{1}, \cdots, u_{n}\right)\right\|>r$;

$*$ and for each $\left(t, u_{0}, u_{1}, \cdots, u_{n}\right) \in\left(J \backslash\left\{t_{1}, \cdots, t_{p}\right\}\right) \times R^{n+1}$ the limits exist

$$
\begin{aligned}
& \lim _{t \rightarrow 0^{+}} F\left(t, u_{0}, u_{1}, \cdots, u_{n}\right)=F\left(0, u_{0}, u_{1}, \cdots, u_{n}\right), \\
& \lim _{t \rightarrow t_{k}^{-}} F\left(t, u_{0}, u_{1}, \cdots, u_{n}\right)=F\left(t_{k}, u_{0}, u_{1}, \cdots, u_{n}\right)
\end{aligned}
$$

and

$$
\begin{aligned}
& \lim _{t \rightarrow t_{k}^{+}} F\left(t, u_{0}, u_{1}, \cdots, u_{n}\right), \quad k=1, \cdots, p, \\
& \lim _{t \rightarrow T^{+}} F\left(t, u_{0}, u_{1}, \cdots, u_{n}\right)=F\left(T, u_{0}, u_{1}, \cdots, u_{n}\right)
\end{aligned}
$$

exist.

By a solution of (9) we mean a function $u \in X$ satisfying (9).

The following abstract existence theorem is also used in this paper, whose proof can be see in [23].

Lemma 2.1. Let $X$ and $Y$ be Banach spaces. Suppose $L: D(L) \subset X \rightarrow Y$ is a Fredholm operator of index zero with $\operatorname{Ker} L=\{0\}, N: X \rightarrow Y$ is $L$-compact on any open bounded subset of $X$. If $0 \in \Omega \subset X$ is a open bounded subset and $L x \neq \lambda N x$ for all $x \in D(L) \cap \partial \Omega$ and $\lambda \in[0,1]$, then there is at least one $x \in \Omega$ so that $L x=N x$.

Now, we define the linear operator $L: D(L) \subseteq X \rightarrow Y$ and the nonlinear operator $N: X \rightarrow Y$ by

$$
L x(t)=\left(\begin{array}{c}
x^{(n)}(t) \\
\Delta x\left(t_{1}\right) \\
\cdot \\
\cdot \\
\Delta x\left(t_{p}\right) \\
\cdot \\
\cdot \\
\Delta x^{(n-1)}\left(t_{1}\right) \\
\cdot \\
\cdot \\
\Delta x^{(n-1)}\left(t_{p}\right)
\end{array}\right) \text { for } x \in D(L)
$$


where $D(L)=\left\{u \in X, u_{k} \in C^{n}\left(t_{k}, t_{k+1}\right), k=0,1, \cdots, m\right\}$ and

$$
N x(t)=\left(\begin{array}{c}
f\left(t, x(t), x^{\prime}(t), \cdots, x^{(n-1)}(t)\right) \\
I_{0,1}\left(x\left(t_{k}\right), x^{\prime}\left(t_{k}\right), \cdots, x^{(n-1)}\left(t_{k}\right)\right) \\
\cdot \\
\cdot \\
I_{0, p}\left(x\left(t_{k}\right), x^{\prime}\left(t_{k}\right), \cdots, x^{(n-1)}\left(t_{k}\right)\right) \\
\cdot \\
\cdot \\
I_{n-1,1}\left(x\left(t_{k}\right), x^{\prime}\left(t_{k}\right), \cdots, x^{(n-1)}\left(t_{k}\right)\right) \\
\cdot \\
\cdot \\
I_{n-1, p}\left(x\left(t_{k}\right), x^{\prime}\left(t_{k}\right), \cdots, x^{(n-1)}\left(t_{k}\right)\right)
\end{array}\right) \text { for } x \in X .
$$

It is easy to see that $L$ is a Fredholm operator of index zero with $\operatorname{Ker} L=\{0\}, N$ is $L$-compact on any open bounded subset of $X$ and that $x \in X$ is a solution of problem (9) if and only if $x$ is a solution of the operator equation $L x=N x$.

We set the following assumptions which should be used in Theorem 2.1.

(A) $I_{n-1, k}\left(x_{0}, \cdots, x_{n-1}\right)\left(2 x_{n-1}+I_{n-1, k}\left(x_{0}, \cdots, x_{n-1}\right) \geq 0\right.$ for all $x \in R$.

(B) There are numbers $\alpha_{i, k} \geq 0$ such that $\left|I_{i, k}\left(x_{0}, \cdots, x_{n-1}\right)\right| \leq \alpha_{i, k}\left|x_{i}\right|$ for all $i=0, \cdots, n-2$ and $k=1, \cdots, p$ with $\sum_{k=1}^{p} \alpha_{i, k}<\frac{1}{2}$ for all $i=$ $0, \cdots, n-2$.

(C) There are functions $h:[0, T] \times R^{n} \rightarrow R$ and $g_{i}:[0, T] \times R \rightarrow R$ such that

(i) $f\left(t, x_{0}, \cdots, x_{n-1}\right)=h\left(t, x_{0}, \cdots, x_{n-1}\right)+\sum_{i=0}^{n-1} g_{i}\left(t, x_{i}\right)$ holds for all $\left(t, x_{0}, \cdots\right.$, $\left.x_{n-1}\right) \in[0, T] \times R^{n}$.

(ii) $g_{i}(t, x)$ satisfies that $g_{i}(\bullet, x) \in X$ for every $x \in R$ and $g_{i}(t, \bullet)$ is continuous for every $t \in[0, T]$.

(iii) $h$ satisfies that $h\left(\bullet, x_{0}, \cdots, x_{n-1}\right) \in C_{p}^{0}$ for every $\left(x_{0}, \cdots, x_{n-1}\right) \in R^{n}$ and $g_{i}(t, \bullet, \cdots, \bullet)$ is continuous for every $t \in[0, T]$.

(iv) There are constants $m \geq 0$ and $\beta>0$ so that

$$
h\left(t, x_{0}, \cdots, x_{n-1}\right) x_{n-1} \geq \beta\left|x_{n-1}\right|^{m+1}
$$

holds for all $\left(t, x_{0}, \cdots, x_{n-1}\right) \in[0, T] \times R^{n}$.

(v) $\lim _{|x| \rightarrow+\infty} \sup _{t \in[0, T]} \frac{\left|g_{i}(t, x)\right|}{|x|^{m}}=r_{i} \in[0,+\infty)$ for $i=0, \cdots, n-1$. 
Theorem 2.1. Suppose $(B),(A)$ and $(C)$ hold. Then problem (9) has at least one solution if

$$
r_{0}+\sum_{k=1}^{n-1} r_{k}\left(\frac{3}{2}\right)^{m(n-k-2)} \prod_{i=k}^{n-2}\left(\frac{1}{1-2 \sum_{k=1}^{p} \alpha_{i, k}}\right)^{m}<\beta .
$$

Proof. Let $\lambda \in(0,1)$. Suppose $x$ is a solution of the system

$$
\left\{\begin{array}{r}
x^{(n)}(t)=\lambda f\left(t, x(t), x^{\prime}(t), \cdots, x^{(n-1)}(t)\right), \\
t \in[0, T], t \neq t_{k}, k=1, \cdots, p, \\
\Delta x^{(i)}\left(t_{k}\right)=\lambda I_{i, k}\left(x\left(t_{k}\right), \cdots, x^{(n-1)}(t)\right), \\
k=1, \cdots, p, i=0, \cdots, n-1, \\
x^{(i)}(0)=-x^{(i)}(T), i=0, \cdots, n-1 .
\end{array}\right.
$$

For $i=0, \cdots, n-2$, from $x^{(i)}(0)=-x^{(i)}(T)$, we get

$$
x^{(i)}(0)=-\frac{1}{2}\left(\int_{0}^{T} x^{(i+1)}(s) d s+\sum_{k=1}^{p} I_{i, k}\left(x\left(t_{k}\right), \cdots, x^{(n-1)}(t)\right)\right) .
$$

Then

$$
\begin{aligned}
\left|x^{(i)}(0)\right| & \leq \frac{1}{2}\left(\int_{0}^{T}\left|x^{(i+1)}(s)\right| d s+\sum_{k=1}^{p}\left|I_{i, k}\left(x\left(t_{k}\right), \cdots, x^{(n-1)}(t)\right)\right|\right) \\
& \leq \frac{1}{2}\left(\int_{0}^{T}\left|x^{(i+1)}(s)\right| d s+\sum_{k=1}^{p} \alpha_{i, k}\left|x^{(i)}\left(t_{k}\right)\right|\right) .
\end{aligned}
$$

So,

$$
\begin{aligned}
\left|x^{(i)}(t)\right| \leq & \left|\int_{0}^{t} x^{(i+1)}(s) d s\right|+\sum_{0<t_{k}<t}\left|I_{i, k}\left(x\left(t_{k}\right), \cdots, x^{(n-1)}(t)\right)\right| \\
& +\frac{1}{2}\left(\int_{0}^{T}\left|x^{(i+1)}(s)\right| d s+\sum_{k=1}^{p} \alpha_{i, k}\left|x^{(i)}\left(t_{k}\right)\right|\right) \\
\leq & \frac{3}{2} \int_{0}^{T}\left|x^{(i+1)}(s)\right| d s+2 \sum_{k=1}^{p} \alpha_{i, k}\left|x^{(i)}\left(t_{k}\right)\right| .
\end{aligned}
$$


We get

$$
\left\|x^{(i)}\right\|_{\infty} \leq \frac{3}{2-4 \sum_{k=1}^{p} \alpha_{i, k}} \int_{0}^{T}\left|x^{(i+1)}(s)\right| d s \text { for } i=0, \cdots, n-2 .
$$

Hence we get

$$
\left\|x^{(i)}\right\|_{\infty} \leq\left(\frac{3}{2}\right)^{n-2-i} \prod_{k=i}^{n-2} \frac{1}{1-2 \sum_{k=1}^{p} \alpha_{i, k}} \int_{0}^{T}\left|x^{(n-1)}(s)\right| d s \text { for } i=0, \cdots, n-2 .
$$

We divide the remainder of the proof into two steps.

Step 1. Prove that there is a constant $M>0$ so that $\int_{0}^{T}\left|x^{(n-1)}(s)\right|^{m+1} d s \leq$ $M$.

Transforming the first equation of (9) to

$$
x^{(n)}(t) x^{(n-1)}(t)=\lambda f\left(t, x(t), x^{\prime}(t), \cdots, x^{(n-1)}(t)\right) x^{(n-1)}(t) .
$$

Integrating it from 0 to $T$, we get

$$
\begin{aligned}
& -\frac{1}{2} \sum_{k=1}^{p}\left[\left(x^{(n-1)}\left(t_{k}^{+}\right)\right)^{2}-\left(x^{(n-1)}\left(t_{k}^{-}\right)\right)^{2}\right] \\
= & \lambda \int_{0}^{T} f\left(s, x(t), x^{\prime}(t), \cdots, x^{(n-1)}(t)\right) x^{(n-1)}(s) d s \\
= & \lambda\left(\int_{0}^{T} h\left(s, x(t), x^{\prime}(t), \cdots, x^{(n-1)}(t)\right) x^{(n-1)}(s) d s+\int_{0}^{T} g_{0}(s, x(s)) x(s) d s\right. \\
& \left.+\sum_{i=1}^{n-1} \int_{0}^{T} g_{i}\left(s, x^{(i)}(s)\right) x^{(n-1)}(s) d s+\int_{0}^{T} r(s) x^{(n-1)}(s) d s\right) .
\end{aligned}
$$

It follows from $(A)$ that

$$
\begin{aligned}
& \left(x^{(n-1)}\left(t_{k}^{+}\right)\right)^{2}-\left(x^{(n-1)}\left(t_{k}^{-}\right)\right)^{2} \\
= & \left(x^{(n-1)}\left(t_{k}^{+}\right)-x^{(n-1)}\left(t_{k}^{-}\right)\right)\left(x^{(n-1)}\left(t_{k}^{+}\right)+x^{(n-1)}\left(t_{k}^{-}\right)\right) \\
= & \Delta x^{(n-1)}\left(t_{k}^{-}\right)\left(2 x^{(n-1)}\left(t_{k}^{-}\right)+\Delta x^{(n-1)}\left(t_{k}^{-}\right)\right) \\
= & I_{k}\left(x\left(t_{k}^{-}\right), \cdots, x^{(n-1)}\left(t_{k}^{-}\right)\right)\left(2 x^{(n-1)}\left(t_{k}^{-}\right)+I_{k}\left(x\left(t_{k}^{-}\right), \cdots, x^{(n-1)}\left(t_{k}^{-}\right)\right)\right) \\
\geq & 0 .
\end{aligned}
$$


So

$$
\begin{aligned}
& \int_{0}^{T} h\left(s, x(t), x^{\prime}(t), \cdots, x^{(n-1)}(t)\right) x^{(n-1)}(s) d s+\int_{0}^{T} g_{0}(s, x(s)) x^{(n-1)}(s) d s \\
& +\sum_{i=1}^{n-1} \int_{0}^{T} g_{i}\left(s, x^{(i)}(s)\right) x^{(n-1)}(s) d s+\int_{0}^{T} r(s) x^{(n-1)}(s) d s \leq 0 .
\end{aligned}
$$

It follows from $(C)$ that

$$
\begin{aligned}
& \beta \int_{0}^{T}\left|x^{(n-1)}(s)\right|^{m+1} d s \\
\leq & -\int_{0}^{T} g_{0}(s, x(s)) x^{(n-1)}(s) d s-\sum_{i=1}^{n-1} \int_{0}^{1} g_{i}\left(s, x^{(i)}(s)\right) x^{(n-1)}(s) d s \\
& -\int_{0}^{T} r(s) x^{(n-1)}(s) d s \\
\leq & \int_{0}^{T}\left|g_{0}(s, x(s))\right|\left|x^{(n-1)}(s)\right| d s+\sum_{i=1}^{n-1} \int_{0}^{T}\left|g_{i}\left(s, x^{(i)}(s)\right)\right|\left|x^{(n-1)}(s)\right| d s \\
& +\int_{0}^{T}|r(s)|\left|x^{(n-1)}(s)\right| d s .
\end{aligned}
$$

Let $\epsilon>0$ satisfy that

$$
\left(r_{0}+\epsilon\right)+\sum_{k=1}^{n}\left(r_{k}+\epsilon\right)\left(\frac{3}{2}\right)^{m(n-k-2)} \prod_{i=k}^{n-2}\left(\frac{1}{1-2 \sum_{k=1}^{p} \alpha_{i, k}}\right)^{m}<\beta
$$

For such $\epsilon>0$, there is $\delta>0$ so that for every $i=0,1, \cdots, n$,

$$
\left|g_{i}(t, x)\right|<\left(r_{i}+\epsilon\right)|x|^{m} \text { uniformly for } t \in[0, T] \text { and }|x|>\delta .
$$

Let, for $i=1, \cdots, n, \Delta_{1, i}=\left\{t: t \in[0, T],\left|x^{(i)}\left(\alpha_{i}(t)\right)\right| \leq \delta\right\}, \Delta_{2, i}=\{t:$ $\left.t \in[0, T],\left|x^{(i)}\left(\alpha_{i}(t)\right)\right|>\delta\right\}, \quad g_{\delta, i}=\max _{t \in[0, T],|x| \leq \delta}\left|g_{i}(t, x)\right|$, and $\Delta_{1}=\{t \in$ $[0, T],|x(t)| \leq \delta\}, \Delta_{2}=\{t \in[0, T],|x(t)|>\delta\}$. Then we get

$$
\begin{aligned}
& \beta \int_{0}^{T}\left|x^{(n-1)}(s)\right|^{m+1} d s \\
\leq & \left.\left(r_{0}+\epsilon\right) \int_{0}^{T}\left|x^{(n-1)}(s)\right|^{m+1} d s+\sum_{k=1}^{n-1}\left(r_{k}+\epsilon\right) \int_{0}^{T} \mid x^{(k)}(s)\right)\left.\right|^{m}\left|x^{(n-1)}(s)\right| d s \\
& +\int_{0}^{T}|r(s)|\left|x^{(n-1)}(s)\right| d s+g_{\delta, 0} \int_{0}^{T}\left|x^{(n-1)}(s)\right| d s+\sum_{k=1}^{n-1} g_{\delta, k} \int_{0}^{T}\left|x^{(n-1)}(s)\right| d s
\end{aligned}
$$




$$
\begin{aligned}
& \leq\left(r_{0}+\epsilon\right) \int_{0}^{T}\left|x^{(n-1)}(s)\right|^{m+1} d s \\
& \left.+\left.\sum_{k=1}^{n}\left(r_{k}+\epsilon\right)\left(\int_{0}^{T} \mid x^{(k)}(s)\right)\right|^{m+1} d s\right)^{m /(m+1)}\left(\int_{0}^{T}\left|x^{(n-1)}(s)\right|^{m+1} d s\right)^{1 /(m+1)} \\
& +\left(\int_{0}^{T}|r(s)| d s\right)^{m /(m+1)}\left(\int_{0}^{T}\left|x^{(n-1)}(s)\right|^{m+1} d s\right)^{1 /(m+1)} \\
& +\sum_{k=0}^{n-1} g_{\delta, k} \int_{0}^{T}\left|x^{(n-1)}(s)\right| d s \\
& =\left(r_{0}+\epsilon\right) \int_{0}^{T}\left|x^{(n-1)}(s)\right|^{m+1} d s \\
& +\sum_{k=1}^{n-1}\left(r_{k}+\epsilon\right)\left(\int_{0}^{T}\left|x^{(i)}(u)\right|^{m+1} d u\right)^{m /(m+1)}\left(\int_{0}^{T}\left|x^{(n-1)}(s)\right|^{m+1} d s\right)^{1 /(m+1)} \\
& +\left(\int_{0}^{T}|r(s)| d s\right)^{m /(m+1)}\left(\int_{0}^{T}\left|x^{(n-1)}(s)\right|^{m+1} d s\right)^{1 /(m+1)} \\
& +T^{m /(m+1)} \sum_{k=0}^{n-1} g_{\delta, k}\left(\int_{0}^{T}\left|x^{(n-1)}(s)\right|^{m+1} d s\right)^{1 /(m+1)} \\
& \leq\left(r_{0}+\epsilon\right) \int_{0}^{T}\left|x^{(n-1)}(s)\right|^{m+1} d s \\
& +\sum_{k=1}^{n-1}\left(r_{k}+\epsilon\right)\left(\int_{0}^{T}\left|x^{(i)}(u)\right|^{1+m} \mid d u\right)^{m /(m+1)}\left(\int_{0}^{T}\left|x^{(n-1)}(s)\right|^{m+1} d s\right)^{1 /(m+1)} \\
& +\left(\int_{0}^{T}|r(s)| d s\right)^{m /(m+1)}\left(\int_{0}^{T}\left|x^{(n-1)}(s)\right|^{m+1} d s\right)^{1 /(m+1)} \\
& +T^{m /(m+1)} \sum_{k=0}^{n-1} g_{\delta, k}\left(\int_{0}^{T}\left|x^{(n-1)}(s)\right|^{m+1} d s\right)^{1 /(m+1)} \\
& =\left(\left(r_{0}+\epsilon\right)+\sum_{k=1}^{n-1}\left(r_{k}+\epsilon\right)\left(\frac{3}{2}\right)^{m(n-k-2)} \prod_{i=k}^{n-2}\left(\frac{1}{1-2 \sum_{k=1}^{p} \alpha_{i, k}}\right)^{m}\right) \int_{0}^{T}\left|x^{(n-1)}(s)\right|^{m+1} d s \\
& +\left(\int_{0}^{T}|r(s)|\right)^{m /(m+1)}\left(\int_{0}^{T}\left|x^{(n-1)}(s)\right|^{m+1} d s\right)^{1 /(m+1)} \\
& +T^{m /(m+1)} M^{1 /(m+1)} \sum_{k=0}^{n-1} g_{\delta, k}
\end{aligned}
$$

It follows from (14) that there is a constant $M>0$ so that $\int_{0}^{T}\left|x^{(n-1)}(s)\right|^{m+1} d s \leq$ $M$. 
Step 2. Prove that there is a constant $M_{1}>0$ so that $\left\|x^{(n-1)}\right\|_{\infty} \leq M_{1}$.

It follows from Step 1 that there is $\xi \in[0, T]$ so that $\left|x^{(n-1)}(\xi)\right| \leq(M / T)^{1 /(m+1)}$.

Case 1. If $t<\xi$, integrating it from $t$ to $\xi$, we get, using $(A)$, that

$$
\begin{aligned}
& \left(x^{(n-1)}(t)\right)^{2}=\left(x^{(n-1)}(\xi)\right)^{2}-\frac{1}{2} \sum_{t \leq t_{k}<\xi}\left[\left(x^{(n-1)}\left(t_{k}^{+}\right)\right)^{2}-\left(x^{(n-1)}\left(t_{k}^{-}\right)\right)^{2}\right] \\
& -\lambda \int_{t}^{\xi} f\left(s, x(s), \cdots, \cdots, x^{(n-1)}(s)\right) x^{(n-1)}(s) d s \\
\leq & (M / T)^{2 /(m+1)}-\lambda \int_{t}^{\xi} f\left(s, x(s), \cdots, \cdots, x^{(n-1)}(s)\right) x^{(n-1)}(s) d s \\
\leq & (M / T)^{2 /(m+1)}-\lambda\left(\int_{t}^{\xi} h\left(s, x(s), \cdots, x^{(n-1)}(s)\right) x^{(n-1)}(s) d s\right. \\
& +\int_{t}^{\xi} g_{0}(s, x(s)) x^{(n-1)}(s) d s \\
& \left.+\sum_{i=1}^{n-1} \int_{t}^{\xi} g_{i}\left(s, x^{(i)}(s)\right) x^{(n-1)}(s) d s+\int_{t}^{\xi} r(s) x^{(n-1)}(s) d s\right) \\
\leq & (M / T)^{2 /(m+1)}-\int_{t}^{\xi} g_{0}(s, x(s)) x^{(n-1)}(s) d s \\
& -\sum_{i=1}^{n-1} \int_{t}^{\xi} g_{i}\left(s, x^{(i)}(s)\right) x^{(n-1)}(s) d s-\int_{t}^{\xi} r(s) x^{(n-1)}(s) d s \\
\leq & (M / T)^{2 /(m+1)}+\int_{0}^{T}\left|g_{0}(s, x(s))\right|\left|x^{(n-1)}(s)\right| d s \\
& \int_{0}^{T}\left|x^{(n-1)}(s)\right|^{m+1} d s+\left(\int_{0}^{T}|r(s)| d s\right)^{m-1} \int_{i=1}^{T}\left|g_{i}\left(s, x^{(i)}(s)\right)\right|\left|x^{(n-1)}(s)\right| d s \\
& +\sum_{i=1}^{T} \int_{0} \\
& +\int_{0}^{n-2}|r(s)|\left|x^{(n-1)}(s)\right| d s \\
& (M / T)^{2 /(m+1)}+\left[\left(r_{0}+\epsilon\right)+\left.\sum_{k=1}^{n-1}\left(r_{k}+\epsilon\right)\left(\frac{3}{2}\right)^{m(n-k-2)}(s)\right|^{m+1} d s\right)^{1 /(m+1)} \\
& \left.\left.\prod^{m}\right)^{m}\right) \times
\end{aligned}
$$




$$
\begin{aligned}
& +\sum_{k=0}^{n-1} g_{\delta, k} T^{m /(m+1)}\left(\int_{0}^{T}\left|x^{(n-1)}(s)\right|^{m+1} d s\right)^{1 /(m+1)} \\
\leq & (M / T)^{2 /(m+1)}+\left[\left(\left(r_{0}+\epsilon\right)+\sum_{k=1}^{n-1}\left(r_{k}+\epsilon\right)\left(\frac{3}{2}\right)^{m(n-k-2)}\right.\right. \\
& \left.\quad \prod_{i=k}^{n-2}\left(\frac{1}{1-2 \sum_{k=1}^{p} \alpha_{i, k}}\right)^{m}\right) M \\
& \left.+\left(\int_{0}^{T}|r(s)| d s\right)^{m /(m+1)} M^{1 /(m+1)}\right]+T^{m /(m+1)} M^{1 /(m+1)} \sum_{k=0}^{n-1} g_{\delta, k} \\
= & : M_{2} .
\end{aligned}
$$

Hence one sees that

$$
\left[x^{(n-1)}(t)\right]^{2} \leq M_{2}, \text { for } t \in[0, \xi] .
$$

This implies $\left[x^{(n-1)}(0)\right]^{2} \leq M_{3}$. So $\left[x^{(n-1)}(T)\right]^{2}=\left[x^{(n-1)}(0)\right]^{2} \leq M_{3}$. For $t \in[\xi, T]$, we have

$$
\begin{aligned}
\left(x^{(n-1)}(t)\right)^{2}= & \left(x^{(n-1)}(T)\right)^{2}-\frac{1}{2} \sum_{\xi \leq t_{k}<t}\left[\left(x^{(n-1)}\left(t_{k}^{+}\right)\right)^{2}-\left(x^{(n-1)}\left(t_{k}^{-}\right)\right)^{2}\right] \\
& -\lambda \int_{t}^{T} f\left(s, x(s), \cdots, x^{(n-1)}(s)\right) x^{(n-1)}(s) d s .
\end{aligned}
$$

Similar to above discussion, we get that there is $M_{4}>0$ so that $\left[x^{(n-1)}(t)\right]^{2} \leq$ $M_{4}$ for $t \in[\xi, T]$. All above discussion implies that there is $M_{1}>0$ so that $\left|x^{(n-1)}(t)\right| \leq M_{1}$. Thus $\left\|x^{(n-1)}\right\|_{\infty} \leq M_{1}$. So

$$
\begin{aligned}
\left|x^{(k)}(t)\right| & \leq\left(\frac{3}{2}\right)^{m(n-k-2)} \prod_{i=k}^{n-2}\left(\frac{1}{1-2 \sum_{k=1}^{p} \alpha_{i, k}}\right)^{m} \int_{0}^{T}\left|x^{(n-1)}(s)\right| d s \\
& \leq\left(\frac{3}{2}\right)^{m(n-k-2)} \prod_{i=k}^{n-2}\left(\frac{1}{1-2 \sum_{k=1}^{p} \alpha_{i, k}}\right)^{m} M_{1} T .
\end{aligned}
$$

Hence there is $M_{0}>0$ so that

$$
\|x\| \leq \max \left\{\|x\|_{\infty}, \cdots,\left\|x^{(n-1)}\right\|_{\infty}\right\} \leq M_{0}
$$

It follows from Lemma 2.1 that equation $L x=N x$ has at least one solution, which is a solution of problem (9). The proof is complete.

We now set the following assumptions which will be used in Theorem 2.2.

$\left(A^{\prime}\right) . I_{n-1, k}\left(x_{0}, \cdots, x_{n-1}\right)\left(2 x_{n-1}+I_{n-1, k}\left(x_{0}, \cdots, x_{n-1}\right) \leq 0\right.$ for all $x \in R$. that

$\left(C^{\prime}\right)$. There are functions $h:[0, T] \times R^{n} \rightarrow R$ and $g_{i}:[0, T] \times R \rightarrow R$ such 
(i) $f\left(t, x_{0}, \cdots, x_{n-1}\right)=h\left(t, x_{0}, \cdots, x_{n-1}\right)+\sum_{i=0}^{n-1} g_{i}\left(t, x_{i}\right)$ holds for all $\left(t, x_{0}\right.$, $\left.\cdots, x_{n-1}\right) \in[0, T] \times R^{n+1}$.

(ii) $g_{i}(t, x)$ satisfies that $g_{i}(\bullet, x) \in X$ for every $x \in R$ and $g_{i}(t, \bullet)$ is continuous for every $t \in[0, T]$.

(iii) $h$ satisfies that $h\left(\bullet, x_{0}, \cdots, x_{n-1}\right) \in X$ for every $\left(x_{0}, \cdots, x_{n-1}\right) \in R^{n}$ and $h(t, \bullet, \cdots, \bullet)$ is continuous for every $t \in[0, T]$.

(iv) There are constants $m \geq 0$ and $\beta>0$ such that

$$
h\left(t, x_{0}, \cdots, x_{n-1}\right) x_{n-1} \leq-\beta\left|x_{n-1}\right|^{m+1}
$$

holds for all $\left(t, x_{0}, \cdots, x_{n-1}\right) \in[0, T] \times R^{n}$.

(v) $\lim _{|x| \rightarrow+\infty} \sup _{t \in[0, T]} \frac{\left|g_{i}(t, x)\right|}{|x|^{m}}=r_{i} \in[0,+\infty)$ for $i=0, \cdots, n-1$.

Theorem 2.2. Suppose $(B),\left(A^{\prime}\right)$ and $\left(C^{\prime}\right)$ hold. Then problem (9) has at least one solution if

$$
r_{0}+\sum_{k=1}^{n} r_{k}\left(\frac{3}{2}\right)^{m(n-k-2)} \prod_{i=k}^{n-2}\left(\frac{1}{1-2 \sum_{k=1}^{p} \alpha_{i, k}}\right)^{m}<\beta .
$$

Proof. The proof is similar to that of Theorem 2.1. We consider system (10), from assumption $\left(A^{\prime}\right)$, it is easy to get

$$
\begin{aligned}
& \left.\int_{0}^{T} h\left(s, x(s), \cdots, x^{(n-1)}(s)\right)\right) x^{(n-1)}(s) d s+\int_{0}^{T} g_{0}(s, x(s)) x^{(n-1)}(s) d s \\
& +\sum_{i=1}^{n} \int_{0}^{T} g_{i}\left(s, x^{(i)}(s)\right) x^{(n-1)}(s) d s+\int_{0}^{T} r(s) x^{(n-1)}(s) d s \geq 0 .
\end{aligned}
$$

The remainder of the proof is similar to that of Theorem 2.1 and is omitted.

\section{EXAMPLES}

In this section, we give two examples, which can not be solved by the results in previous papers, to illustrate the main results.

Example 3.1. Consider the problem

$$
\left\{\begin{aligned}
x^{(n)}(t)= & a\left[x^{(n-1)}(t)\right]^{2 m+1}+\sum_{k=0}^{n-1} p_{k}(t)\left[x^{(k)}(t)\right]^{2 m+1}+r(t), \\
t \in[0, T], t \neq t_{k}, k=1, \cdots, p & \\
\Delta x^{(i)}\left(t_{k}\right)= & \alpha_{i, k} x^{(i)}\left(t_{k}\right), k=1, \cdots, p, i=0, \cdots, n-1 \\
x^{(i)}(0) & =-x^{(i)}(T), i=0, \cdots, n-1
\end{aligned}\right.
$$


then (16) has at least one solution if

$$
\left\{\begin{array}{l}
a>0, \\
\sum_{k=1}^{p} \alpha_{i, k}<\frac{1}{2}, i=0, \cdots, n-2, \\
\alpha_{n-1, k}\left(2+\alpha_{n-1, k}\right) \geq 0, k=1, \cdots, p \\
\left\|p_{0}\right\|_{\infty}+\sum_{k=1}^{n-1}\left(\frac{3}{2}\right)^{m(n-k-2)} \prod_{i=k}^{n-2}\left(\frac{1}{1-2 \sum_{k=1}^{p} \alpha_{i, k}}\right)^{m}\left\|p_{k}\right\|_{\infty}<a .
\end{array}\right.
$$

Example 3.2. Consider the problem

$$
\left\{\begin{aligned}
x^{(n)}(t)= & a\left[x^{(n-1)}(t)\right]^{2 m+1}+\sum_{k=0}^{n-1} p_{k}(t)\left[x^{(k)}(t)\right]^{2 m+1}+r(t), \\
t \in[0, T], t \neq t_{k}, k=1, \cdots, p & \\
\Delta x^{(i)}\left(t_{k}\right)= & \alpha_{i, k} x^{(i)}\left(t_{k}\right), k=1, \cdots, p, i=0, \cdots, n-1, \\
x^{(i)}(0)= & -x^{(i)}(T), i=0, \cdots, n-1 .
\end{aligned}\right.
$$

Then (17) has at least one solution if

$$
\left\{\begin{array}{l}
\begin{array}{l}
a<0, \\
p
\end{array} \\
\sum_{k=1}^{n} \alpha_{i, k}<\frac{1}{2}, i=0, \cdots, n-2, \\
\alpha_{n-1, k}\left(2+\alpha_{n-1, k}\right) \leq 0, k=1, \cdots, p \\
\left\|p_{0}\right\|_{\infty}+\sum_{k=1}^{n-1}\left(\frac{3}{2}\right)^{m(n-k-2)} \prod_{i=k}^{n-2}\left(\frac{1}{1-2 \sum_{k=1}^{p} \alpha_{i, k}}\right)^{m}\left\|p_{k}\right\|_{\infty}<-a .
\end{array}\right.
$$

\section{REFERENCES}

1. I. T. Kiguradze, T. Kusanno, Periodic solutions of non-autonomous ordinary differential equations of higher order, Differential Equations, 35(1) (1999), 70-77.

2. I. T. Kiguradze, B. Puza, On periodic solutions of systems of differential equations with deviating arguments, Nonlinear Analysis, 42 (2000), 229-242.

3. F. Cong, Periodic solutions for $2 \mathrm{kth}$ order ordinary differential equations with resonance, Nonlinear Analysis, 32 (1998), 787-793.

4. F. Cong, Existence of periodic solutions of $(2 \mathrm{k}+1)$ th order ordinary differential equations, Appl. Math. Letters, 17 (2004), 727-732. 
5. F. Cong, Q. Huang, S. Shi, Existence and uniqueness of solutions for $(2 \mathrm{n}+1)$ th order differential equations, J. Math. Anal. Appl., 241 (2000), 1-9.

6. S. Srzednicki, On periodic solutions of certain $n^{\text {th }}$ differential equations, J. Math. Anal. Appl., 196 (1995), 666-675.

7. I. T. Kigaradze, On periodic solutions of nth order ordinary differential equations, Nonlinear Analysis, 40 (2000), 309-321.

8. D. Franco, J. J. Nieto, First order impulsive ordinary differential equations with anti-periodic and nonlinear boundary conditions, Nonl. Anal., 42 (2000), 163-173.

9. Y. Q. Chen, On Massera's theorem for anti-periodic solution, Adv. Math. Sci. Aool., 9 (1999), 125-128.

10. Y. Yin, Remarks on first order differential equations with anti-periodic boundary conditions, Nonlinear Times Digest, 2 (1995), 83-94.

11. D. Franco, J. J. Nieto, D. O’Regan, Anti-periodic boundary value problems for nonlinear first order ordinary differential equations, Math. Inequal. Appl., 6 (2003), 477-485.

12. W. Ding, M. Han and J. Mi, Periodic boundary value problem for the secondorder impulsive functional differential equations, Computers and Mathematics with Applications, 50 (2005), 491-507.

13. J. J. Nieto and R. Rodriguez-Lopez, Periodic boundary value problem for nonLipschitzian impulsive functional differential equations, J. Math. Anal. Appl., 318 (2006), 593-610.

14. W. Ding, J. Mi and M. Han, Periodic boundary value problems for the first order impulsive functional differential equations, Applied Mathematics and Computation, 165 (2005), 433-446.

15. W. Ding and M. Han, Periodic boundary value problem for the second order impulsive functional differential equations, Applied Mathematics and Computation, 155 (2004), 709-726.

16. Z. Luo, J. Shen and J. Nieto, Antiperiodic boundary value problem for first-order impulsive ordinary differential equations, Computers and Mathematics with Applications, 49 (2005), 253-261.

17. Y. Chen, X. Wang and H. Xu, Anti-periodic solutions for semilinear evolution equations, Journal of Mathematical Analysis and Applications, 273 (2002), 627-636.

18. S. Aizicovici, M. McKibben and S. Reich, Anti-periodic solutions to nonmonotone evolution equations with discontinuous nonlinearities, Nonlinear Analysis, 43 (2001), 233-251.

19. A. R. Aftabizadeh, S. Aizicovici and N. H. Pavel, On a class of second-order antiperiodic boundary value problems, Journal of Mathematical Analysis and Applications, 171 (1992), 301-320. 
20. A. R. Aftabizadeh, S. Aizicovici and N. H. Pavel, Anti-periodic boundary value problems for higher order differential equations in Hilbert spaces, Nonlinear Analysis, 18 (1992), 253-267.

21. D. Franco and J. J. Nieto, First-order impulsive ordinary differential equations with anti-periodic and nonlinear boundary conditions, Nonlinear Analysis, 42 (2000), 163173.

22. A. R. Aftabizadeh, Y. K. Huang and N. H. Pavel, Nonlinear Third-Order Differential Equations with Anti-periodic Boundary Conditions and Some Optimal Control Problems, Journal of Mathematical Analysis and Applications, 192 (1995), 266-293.

23. R. E. Gaines and J. L. Mawhin, Coincidence Degree and Nonlinear Differential Equations, Lecture Notes in Math. 568, Springer, Berlin, 1977.

24. C. Ahn, C. Rim, Boundary flows in general coset theories, J. Physics, A, 32 (1999), 2509-2525.

25. S. Pinsky, U. Trittmann, Anti-periodic boundary conditions in supersymmetric discrete light cone quantization, Physics Rev. D3, 62 (2000).

26. H. Kleinert, Functional determinants from Wronski Green functions, J. Math. Phys., 40 (1999), 6044-6051.

27. V. Lakshmikantham, D. D. Bainov, P. S. Simenov, Theory of impulsive differential equations, Singapore, World Scientific, 1989.

28. S. Aizicovici and N. H. Pavel, Anti-periodic solutions to a class of nonlinear differential equations in Hilbert space, Journal of Functional Analysis, 99 (1991), 387-408.

29. Y. Chen, Anti-periodic solutions for semilinear evolution equations, J. Math. Anal. Appl., 315 (2006), 337-348.

30. Y. Chen, Y. J. Cho and D. O'Regan, Anti-periodic solutions for evolution equations with mappings in the class $S_{+}$, Math. Nachr., 278 (2005), 356-362.

31. A. M. Samoilenko and N. A. Perestyuk. Impulsive Differential Equations, World Scientific, Singapore, 1995.

32. S. T. Zavalishchin and A. N. Sesekin, Dynamic impulsive systems, Theory and Applications, Kluwer Acad. Pub., Dordrecht, 1997.

33. Y. V. Rogovchenko. Impulsive evolution systems: Main results and new trends, Dyman. Contin. Discrete Impuls. Systems, 3 (1997), 57-88.

34. D. Qian and X. Li. Periodic solutions for ordinary differential equations with sublinear impulsive effects, J. Math. Anal. Appl., 303 (2005), 288-300.

35. J. M. Belley and M. Virgilio, Periodic Linard-type delay equations with state-dependent impulses, Nonlinear Anal., 64 (2006), 568-589.

36. J. Li and J. Shen, Periodic boundary value problems for delay differential equations with impulses, J. Comput. Appl. Math., 193 (2006), 563-573. 
37. W. Zhang and M. Fan, Periodicity in a generalized ecological competition system governed by impulsive differential equations with delays, Math. Comput. Model., 39 (2004), 479-49.

38. J. Yan, A. Zhao and J.J. Nieto, Existence and global attractivity of positive periodic solution of periodic single-species impulsive Lotka-Volterra systems, Math. Comput. Model., 40 (2004), 509-518.

39. W. Li and H. Huo. Global attractivity of positive periodic solutions for an impulsive delay periodic model of respiratory dynamics, J. Comput. Appl. Math., 174 (2005), 227-238.

40. S. Tang and L. Chen. Density-dependent birth rate, birth pulses and their population dynamic consequences, J. Math. Biol., 44 (2002), 185-199.

41. A. d'Onofrio, On pulse vaccination strategy in the SIR epidemic model with vertical transmission, Appl. Math. Lett., 18 (2005), 729-32.

42. S. Gao, L. Chen, J. J. Nieto and A. Torres, Analysis of a delayed epidemic model with pulse vaccination and saturation incidence, Vaccine, 24 (2006), 6037-6045.

43. S. Tang, and L. Chen. Density-dependent birth rate, birth pulses and their population dynamic consequences, J. Math. Biol., 44 (2002), 185-199.

44. X. Zhang, Z. Shuai and K. Wang, Optimal impulsive harvesting policy for single population, Nonlinear Anal. Real World Applications, 4 (2003), 639-651.

\author{
Yuji Liu \\ Department of Mathematics, \\ Guangdong University of Business Studies, \\ Guangzhou, 510000, \\ P. R. China \\ E-mail: liuyuji888@sohu.com \\ Zhanji Gui \\ Department of Computer Science and Educational Techniques, \\ Hainan Normal University, \\ Haikou, 510000, \\ P. R. China
}

